\title{
SURFACE KNOWLEDGE: TOWARD A PREDICTIVE THEORY OF MATERIALS
}

\author{
To understand how materials function, we must understand how molecules from the \\ environment interact with their surfaces. This article describes new methodologies for \\ studying surface dynamics at the atomic level over a range of time scales.
}

\begin{abstract}
S urfaces are the cutting edge of materials science. Molecules from the environmental gas or liquid phase come in contact with a material at its surface, and chemical bonds of these approaching molecules are cut and new bonds formed. To understand how materials function, and how to produce and enhance them, we must understand surfaces.

We focus here on advances in this field, specifically, the development of methodologies that combine density-functional theory with elasticity theory, thermodynamics, or statistical mechanics. The resulting approaches, while computationally elaborate, let us treat thousands of atoms to follow their wanderings and interplay over time scales from picoseconds up to seconds. Most important, the methods let us analyze the results and gain much insight into how surfaces function.

The examples we discuss relate to semiconductor growth and nanotechnology, but the approaches have much wider application: Researchers are using analogous methods to model the growth of thin magnetic metal films, ${ }^{1}$ catalysis, and corrosion. ${ }^{2}$
\end{abstract}

1521-9615/01/\$10.00 ๑ 2001 IEEE

Peter Kratzer and Matthias Scheffler

Fritz-Haber-Institut der Max-Planck-Gesellscbaft

\section{Advancing materials science}

Materials science seeks to design new materials, improve the quality of existing materials, and make the production process more efficientthat is, cheaper. Current experimental methods to develop new materials still use trial and error. Theoretical modeling of technologically relevant chemical processes still employs mostly phenomenological methods (such as rate equations or hydrodynamic theories) together with "effective" parameters. These parameters typically have limited physical meaning and apply only within narrow temperature, pressure, or material composition limits. Some researchers refer to this as "modeling without microscopic understanding." Despite the term's negative slant, this approach has proven the most valuable to date.

Clearly, however, we need improved methods, and we posit that the next step in basic research involves developing a theoretical approach "with microscopic understanding." This article describes our progress in and perception of developing such a predictive theory of materials.

\section{Challenges}

Understanding material surface properties has become critical to improving the technology on which our modern life is built. For example, semiconductor production involves depositing 
thin layers of a semiconductor, insulator, or metal, then structuring and processing these films with various techniques. Molecular modeling can advance our atomistic understanding and hence implementation of these processes.

We stress general principles of molecular modeling by focusing on two examples:

1. deposition of modern compound semiconductors, such as GaAs and InAs, and

2. spontaneous structure formation in these systems, the so-called self-assembly of nanoscale islands.

If these islands are embedded in a matrix of semiconducting material, covering them with a capping layer, they can be used as quantum dots. These nanoscale structures confine charge carriers (electrons or holes) due to quantum-mechanical effects. Researchers already use them for light-emitting diodes, lasers, and single-electron transistors, and future applications might include quantum computing. ${ }^{3}$

Modeling thin film deposition requires describing a sample area of at least mesoscopic size, say $1000 \mathrm{~nm}^{2}$, involving several thousands of atoms. The time scale the simulation covers should correspond with the actual time needed to deposit one atomic layer; that is, seconds.

However, the atomistic processes that govern the physics and chemistry of deposition, adsorption, and diffusion operate in the length and time domains of 0.1 to $1 \mathrm{~nm}$ and femto- to picoseconds. To model film growth and incorporate information from the atomistic properties, therefore, we must cover huge length scales and time scales: from $10^{-10}$ to $10^{-6} \mathrm{~m}$ and from $10^{-15}$ to $10^{0}$ seconds. Figure 1 shows the range of scales involved.

Smaller length scales of a few hundred atoms typically suffice to discern, for example, the atomic structure of a step on a surface and its role for chemical reactions and atom diffusion. To produce trustworthy molecular modeling, however, we must address the gap between the atomic and the practically relevant time scales, and the crucial role of statistical mechanics.

Simulating crystal growth requires covering large time scales because often rare events rule the whole scenario. The events are rare because some atomistic effects are thermally activated in materials processing and occur with an exponentially small probability yet might ignite important, possibly crucial follow-up surface modifications. The rare-event problem precludes the use

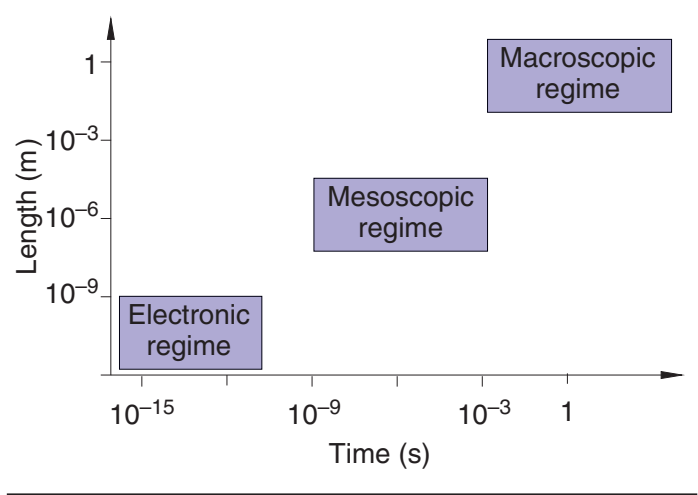

Figure 1. Molecular modeling on the basis of firstprinciples electronic structure calculations requires covering the length and time scales from the electronic to the mesoscopic or even macroscopic.

of straightforward (brute force) molecular-dynamics (MD) techniques (both empirical and, even more so, ab initio MD) to simulate thermally activated processes at realistic temperatures.

Researchers have devised special MD techniques to alleviate this difficulty, ${ }^{4}$ but even with these techniques only certain problems in statistical mechanics are accessible to MD simulations. The interplay and interdependence between different molecular processes presents an even more severe-and not often apparent-problem. A process' importance, measured as how often it occurs during a given time interval, relates not just to its intrinsic rate but also to other processes required to create, replenish, or prolong the lifetime of an important precursor configuration.

Such interdependencies might emerge only after fairly long simulations-perhaps milliseconds (as in the example of GaAs growth discussed later)and after extensive sampling of the system's phase space, yet they significantly affect the simulation's outcome. In our example of film growth, the morphology of the growing film would be affected. We will show here how combining density-functional theory (DFT) with kinetic Monte Carlo (kMC) simulations offers an efficient and accurate way to access sufficiently long time scales.

While many aspects of materials science and molecular modeling require bridging length and time scales, the systems we focus on here have an important similarity that facilitates the treatment and that we consequently exploit: During adsorption, diffusion, and epitaxial growth, the material retains its basic crystalline structure. This lets us assign a crystal lattice to the atoms' possible stable or metastable positions, while (necessarily) including atomic displacements 
away from the lattice sites. Such atomic displacements result from strain fields or the absence of a full coordination shell of neighbors, such as near a step edge or defect, or occur as a (maybe local) surface reconstruction.

The simulation's reliability and computational cost depend on the quality level chosen to describe the elementary processes - that is, how we treat the electrons and the atom-atom interactions. Traditional approaches have been empirical-for example, bond-strength-bond-order potentials or even simpler bonding descriptions. When chemical bonds between atoms break or new bonds form, however, we need a description that accounts for the electrons' quantum-mechanical nature. This applies to surfaces in general (since bonds present in the bulk had to be broken to create the surface), and even more so to chemical reactions occurring on them.

Recent work shows considerable progress in understanding surfaces and surface chemical reactions using ab initio methods, particularly using DFT for total-energy calculations. ${ }^{5}$ Analogous to the multiplicity of scales shown in Figure 1, we propose a multistep approach:

1. We perform DFT calculations for possibly complex system aspects that don't require knowledge of the full size of the system being modeled. Examples include a particular facet of a crystal, or a set of atomic configurations that occur during growth. Usually, before modeling can begin, we must collect data from many such DFT calculations in a database.

2. Using this database, we run code that performs the simulation-that is, treats the thermodynamics or statistical mechanics.

3. We propose a learning approach for future work, starting with an approximate description of the atomic interactions by analytic potentials. This will let us explore a huge number of molecular processes with modest computational effort, perhaps by using some special accelerated MD technique. As the simulation proceeds, we identify the important processes to be calculated with improved accuracy using DFT, then use these DFT results to improve the database obtained from the initial (semi-empirical) description.

\section{Density-functional theory calculations}

To describe bonding in a solid or at its surface, we must calculate its electronic structure, which implies a quantum-mechanical description of the many-electron problem. ${ }^{5}$ In computational terms, electronic degrees of freedom and their associated length scale come into play, which can make simulations costly. DFT represents an efficient approach to describe quantum effects in the electronic structure of a polyatomic system.

This approach is exact in principle, but despite DFT's remarkable success in the past decade, we must remember that in daily practice we are working with approximate density functionals, whose performance we must assess for each system at hand. Once we've selected an appropriate density functional, a DFT calculation of the electronic structure amounts to solving separate Schrödinger-like equations for all electrons in the system, with an additional potential accounting for the electrons' many-particle (exchange and correlation) effects.

Although they substantially simplify the numerical task compared to many-particle methods, DFT calculations still require much data handling because during the calculation we must store and access the information pertinent to the wave functions of all electrons in the problem. The computationally most straightforward way of representing the (complex-valued) wave functions is to store them on a grid, either in real space or by storing their Fourier coefficients. Many computers offer efficient fast Fourier transform routines that permit switching between wave function representations in real space and in reciprocal (wave-vector) space. These algorithms form the heart of modern electronic structure codes.

So far, we've discussed computer codes using the pseudopotential-plane-wave method, which explicitly treats only the atoms' valence shell electrons. This method creates efficient codes that permit calculations on several hundred atoms on parallel architectures. ${ }^{6}$ Methods for solving the full problem for all electrons numerically, such as the linearized augmented plane-wave (LAPW) method, are also available and have also been ported to parallel computers, ${ }^{7,8}$ but the higher demands they place on computer resources to describe the entire electronic system restricts them to problems involving fewer atoms, presently up to about 100 .

Figure 2 illustrates how we use DFT calculations relevant to the modeling of island nucleation and crystal growth by molecular beam epitaxy (MBE). We model a crystal surface by repeating the group of atoms shown in Figure 2 periodically. Figure 2 a shows a cut perpendicular to the 


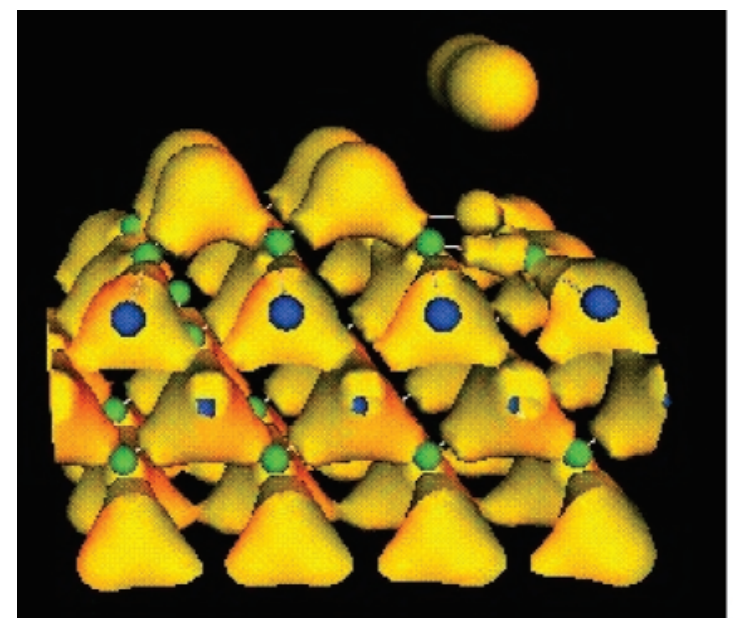

(a)

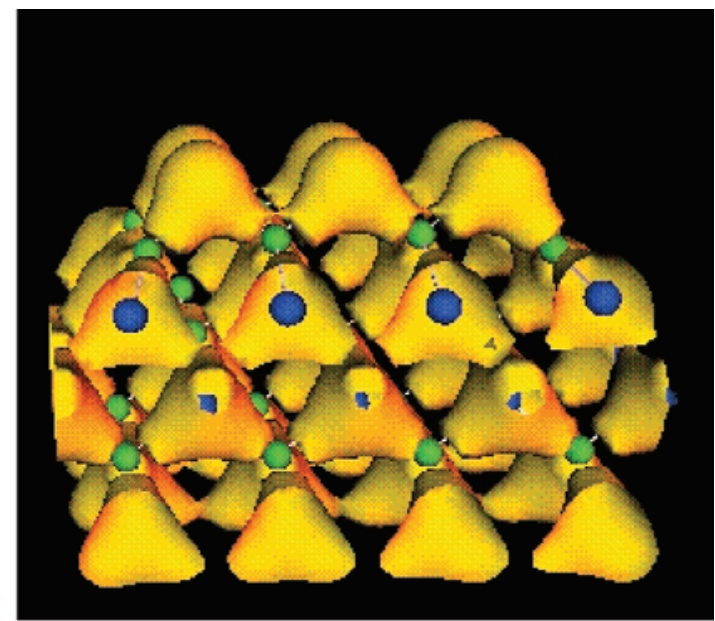

(b)

Figure 2. DFT electronic-structure calculation for the adsorption of an $\mathrm{As}_{2}$ molecule on the $\mathrm{GaAs}(001)$ surface. In (a), the $A s_{2}$ molecule hovers above the surface (yellow balls in the upper part of the picture). In (b), the molecule has adsorbed onto the surface and established chemical bonds with the surface $\mathrm{Ga}$ atoms. The closed yellow surfaces represent contours of constant electron density. The blue dots represent the As nuclei; the green dots, the $\mathrm{Ga}$ atoms.

surface in side view, with As atoms displayed in blue and $\mathrm{Ga}$ atoms in green. The yellow/orange contour surface indicates the valence electron density. Closed shells of electron density surround the As anions, while the Ga cations are depleted of electronic charge. Upon adsorption (Figure $2 \mathrm{~b}$ ), the $\mathrm{As}_{2}$ molecule's electron density agglomerates with the substrate's electron density. We offer a more detailed animation of this process at www.fhi-berlin.mpg.de/th/publications/img/ ie-as2-alpha.gif.

Figure 2 shows an $\mathrm{As}_{2}$ molecule adsorbing on the $\mathrm{GaAs}(001)$ surface where some Ga atoms were deposited before. Surface scientists refer to this surface as being locally in the $\alpha$-reconstruction, a state characterized by bonds between two surface $\mathrm{Ga}$ atoms (a feature absent in the bulk). Adsorption of the $\mathrm{As}_{2}$ molecule breaks the Ga-Ga bonds while As-Ga bonds form simultaneously between the molecule and the surface. The DFT calculation lets us monitor this process by inspecting the electron density at each stage of the approaching $\mathrm{As}_{2}$ molecule. In this way we gain insight into the nature of chemical reactions occurring at the surface. This knowledge, together with the calculated total energies, forms part of the input for using statistical mechanics to understand growth.

\section{Modeling island nucleation}

While the DFT calculations just described consider zero temperature and pressure, model- ing of thin film deposition must treat finite temperature and pressure, and we must even go beyond thermodynamic equilibrium theory: A flux of deposited atoms or molecules implies we are dealing with an open system; we therefore need a kinetic description of molecular processes such as adsorption, desorption, diffusion, and eventually island nucleation and growth.

Our approach exploits the fact that we can represent the atomic configurations that occur during growth on a crystal lattice. We thus replace the physical (Newtonian) surface atom dynamics with a "discrete dynamics": The atoms can move from one lattice site to another or appear on and disappear from the surface (adsorption and desorption) by discrete jumps; after each jump, the system equilibrates in the new geometry before a transition to another state occurs as a thermal fluctuation. Thus, at the temperatures used for the film deposition, these jumps constitute rare events in the sense described earlier.

By concentrating on the jumps, we eliminate the (mostly) superficial information about atom dynamics between rare events- that is, the discrete states' vibrations and the associated time scale. We don't neglect this information but rather describe it in terms of a probability prefactor, discussed later. Each discrete event enters in the simulation with a rate we can determine rigorously to account for the dynamics on the eliminated time scale.

We can demonstrate this for a simple exam- 
ple: a single particle's surface wanderings. ${ }^{9}$ On the atomistic level, the particle's motion is governed by the potential-energy surface (PES), which is the potential energy experienced by the diffusing adatom

$$
\begin{aligned}
& E^{\mathrm{PES}}\left(X_{\mathrm{ad}}, Y_{\mathrm{ad}}\right)= \\
& \min _{Z_{\mathrm{ad}},\left\{R_{I}\right\}} E^{\mathrm{tot}}\left(X_{\mathrm{ad}}, Y_{\mathrm{ad}}, Z_{\mathrm{ad}},\left\{\mathbf{R}_{I}\right\}\right),
\end{aligned}
$$

where $E^{\text {tot }}\left(X_{\text {ad }}, Y_{\text {ad }}, Z_{\text {ad }},\left\{\mathbf{R}_{I J}\right\}\right)$ is the ground-state energy of the many-electron system (also referred to as the total energy) at the atomic configuration $\left(X_{\mathrm{ad}}, Y_{\mathrm{ad}}, Z_{\mathrm{ad}},\left\{\mathbf{R}_{I}\right\}\right)$. The PES represents the minimum total energy with respect to the $z$-coordinate of the adatom $Z_{\mathrm{ad}}$ and all coordinates of the substrate atoms $\left\{\mathbf{R}_{I}\right\}$. If we disregard vibrational contributions to the free energy for a moment, the minima of the potential-energy surface represent the adatom's stable and metastable sites.

The kinetic description of growth defines the underlying atomistic processes, such as diffusion or desorption, in terms of the probabilities with which they occur. Under rather mild assumptions usually fulfilled for processes at a surface, for the rate $\Gamma_{\mathcal{f}}$ of a molecular process $\mathcal{f}$ (diffusion, for example), the rate law reads

$$
\Gamma_{\mathcal{f}}=\frac{k_{\mathrm{B}} T}{b} \exp \left(-\Delta F_{\mathcal{f}} / k_{\mathrm{B}} T\right)
$$

where $\Delta F_{\mathcal{f}}$ is the difference in the Helmholtz free energy between the maximum (saddle point) and the minimum (initial geometry) of the PES along the reaction path of the process 7 . $T$ is the temperature, $k_{\mathrm{B}}$ the Boltzmann constant, and $b$ the Planck constant. The following equation gives the free energy of activation $\Delta F_{\mathcal{f}}$ that the system needs to move from the initial position to the saddle point:

$$
\Delta F_{\mathcal{f}}=\Delta U_{\mathcal{f}}-T \Delta S_{\mathcal{f}}^{\mathrm{vit}} .
$$

The internal energy $U$ in this expression consists of the (static) total energy $E^{\mathrm{PES}}$ and the vibrational energy $U^{\mathrm{vib}}$. $\Delta U_{\mathcal{f}}$ is the difference of the system's internal energy with the particle at the saddle point and at the minimum belonging to process $\mathcal{F}$, and $\Delta S_{\mathcal{J}}^{\text {vib }}$ is the analogous difference in the vibrational entropy. We cast the rate of the process 7 as follows:

$$
\Gamma_{\mathcal{f}}=\Gamma_{\mathcal{f}}^{0} \exp \left(-\Delta E_{\mathcal{f}} / k_{\mathrm{B}} T\right),
$$

where

$$
\Gamma_{\mathcal{f}}^{0}=\left(k_{B} T / h\right) \exp \left(\Delta S_{\mathcal{f}}^{\mathrm{vib}} / k_{\mathrm{B}}-\Delta U_{\mathcal{f}}^{\mathrm{vib}} / k_{\mathrm{B}} T\right),
$$

represents the attempt frequency.

We obtain both basic quantities in Equation $4, \Gamma_{\mathcal{f}}^{0}$ and $\Delta E_{\mathcal{f}}$, from DFT calculations. For single-particle diffusion, we can read $\Delta E_{\mathcal{f}}$ directly from the PES. In this case, we find that $\Gamma_{\mathcal{F}}^{0}$ is of the same order, roughly $10^{13} \mathrm{~s}^{-1}$, for various processes. As a first approximation, we can thus often avoid recalculating $\Gamma_{\mathcal{f}}^{0}$ for each process, because variations in $\Gamma_{\mathcal{F}}^{0}$ by a factor of 5 are typically irrelevant compared to variations of $\Delta E_{\mathrm{J}}$ in the exponent. However, when many atoms are moving in a collective manner, the prefactor might be significantly different. For processes involving several particles, such as nucleation, attachment to islands, or motion along the atomic step that marks an island edge, we must account for the interactions between particles. We can also access these quantities with DFT calculations by calculating the PES, or parts thereof, for all microscopic situations that occur during growth.

However, this involves performing calculations for many different atomic arrangements. The near-sightedness of nature substantially reduces the workload: We can usually describe a surface atom's local chemical bonding by considering a local environment of its nearest and next-nearest neighbors, provided we properly consider the boundary conditions. This nearsightedness reduces a simulation to a recurrence of a possibly large but finite number of local configurations.

To obtain these configurations' binding energies and barriers, we perform DFT calculations and collect the results. Next, a computer program performing a kinetic Monte Carlo growth simulation reads the barriers from a file and calculates a list of rates according to Equation 4. Each simulation step selects a particular process randomly with a probability proportional to its contribution to the total rate. The simulation time advances by an increment given by the inverse total rate multiplied by a random number drawn from an exponentially decaying probability distribution.

This establishes a physically meaningful mapping between simulation time and physical time, thereby exploiting the fact that the microscopic processes eligible within an atomistic time interval can be treated as independent Poissonian processes. ${ }^{10}$ A growth simulation consists of billions of such simulation steps. 
Introducing a lattice-gas Hamiltonian ${ }^{11,12}$ lets us conveniently represent the atomic interaction data obtained from DFT calculations. We discuss this approach schematically for a specific system, homoepitaxy of GaAs by MBE from atomic $\mathrm{Ga}$ and molecular $\mathrm{As}_{2}$ sources on the $\mathrm{GaAs}(001)$ substrate. Under usual MBE growth conditions, the $\mathrm{GaAs}(001)$ surface displays a surface reconstruction terminated by pairs of As dimers, which alternate with "trenches" running in the [110] direction. These trenches stem from a missing pair of As dimers in the surface layer and two missing Ga atoms per unit cell in the layer below.

MBE experiments on GaAs(001) and DFT calculations ${ }^{13,14}$ show that arsenic molecules and gallium atoms interact quite differently with this surface. While $\mathrm{Ga}$ atoms adsorb with unit sticking probability, $\mathrm{As}_{2}$ molecules stick to the surface only after Ga deposition. Surface diffusion distributes the deposited Ga atoms. Growth depends on Ga mobility because strong $\mathrm{As}_{2}$ chemisorption sites only arise after a suitable local arrangement of $\mathrm{Ga}$ adatoms. Once such a site forms, the $\mathrm{As}_{2}$ molecules can adsorb without dissociation and become part of the surface in the form of As dimers. Conversely, As dimers can leave the surface by thermally activated desorption.

To describe As dimers' binding energy at the reconstructed surface and $\mathrm{Ga}$ adatom binding at various sites, we propose a lattice-gas Hamiltonian that consists of a single-site contribution $H_{0}$ plus an interaction term:

$$
H=H_{0}+H_{\text {int }} \text {. }
$$

Let $k$ be a shorthand notation for a lattice site and $n_{k}$ a discrete variable that takes on the value 1 if an As dimer occupies the site or 0 if it's empty. Similarly, $m_{k}$ is an occupation variable describing the $\mathrm{Ga}$ adatoms. Then $H_{0}$ takes on the form

$$
\begin{aligned}
H_{0}= & E_{b}^{\mathrm{As}} \sum_{k} n_{k}+E_{b}^{\mathrm{Ga}} \sum_{k} m_{k} \\
& +V^{\mathrm{Ga}-\mathrm{As}} \sum_{k} n_{k} m_{k}
\end{aligned} .
$$

The reconstructed GaAs(001) surface actually presents some complexity because of the need to distinguish several inequivalent lattice sites due to the reconstruction. To clarify the basic idea, we leave these complications aside for the moment. We describe deviations of the energetics from the single-site values due to different reconstructions and different possible arrangements of $\mathrm{Ga}$ adatoms in the interaction part of the lattice-gas Hamiltonian,

$$
\begin{aligned}
H_{\text {int }}= & \frac{1}{2} V_{1 n}^{\mathrm{As}} \sum_{k, a} n_{k} n_{k+a}+\frac{1}{2} V_{2 n}^{\mathrm{As}} \sum_{k, b} n_{k} n_{k+b} \\
& +\frac{1}{2} V_{1 n}^{\mathrm{Ga}} \sum_{k, a} m_{k} m_{k+a}+\frac{1}{2} V_{2 n}^{\mathrm{Ga}} \sum_{k, b} m_{k} m_{k+b} \\
& +\frac{1}{3} V_{\text {trio }}^{\mathrm{Ga}} \sum_{k, a, a^{\prime}} m_{k} m_{k+a} m_{k+a^{\prime}}+\ldots
\end{aligned}
$$

Here, the indices $k+a$ and $k+b$ denote nearest and next-nearest neighbor sites relative to $k$. DFT calculations performed for a set of atomic configurations determine the interaction parameters

$V_{1 n}^{\mathrm{As}}, V_{2 n}^{\mathrm{As}}, V_{1 n}^{\mathrm{Ga}}, V_{2 n}^{\mathrm{Ga}}$, and $V_{\text {trio }}^{\mathrm{Ga}}$.

Adding more interaction types further refines the description by the lattice-gas Hamiltonian, if required.

To set up a $\mathrm{kMC}$ simulation, we need the binding energies as well as the energy barriers to calculate the rates. These barriers show considerable diversity because each specific process, from an initial state with a given local environment through a transition state to a final state with a different local environment, requires a specific barrier. We must be careful not to violate the principle of detailed balance. This fundamental principle of statistical mechanics requires that the quotient of any forward and backward process rates, obtained by interchanging initial and final states, equals

$$
\Gamma^{(f i)} / \Gamma^{(i f)}=\exp \left(-\Delta F / k_{\mathrm{B}} T\right),
$$

where $\Delta F$ shows how the Helmholtz free energy differs in the initial and final state (or the Gibbs free energy, if desorption into the gas phase is involved).

Figure 3 shows what we can learn from $\mathrm{kMC}$ simulations, specifically, a DFT $+\mathrm{kMC}$ simulation of GaAs homoepitaxy on $\mathrm{GaAs}(001)$ at $T=700 \mathrm{~K}$, Ga flux 0.1 monolayers $/ \mathrm{s}$, and $\mathrm{As}_{2}$ pressure $p=$ $0.85 \times 10^{-5} \mathrm{mbar}$. It shows a small part of the total simulation area, with a "trench" typical for the GaAs surface reconstruction in the center. Ga and As substrate atoms appear in green and dark blue, Ga adatoms in yellow, and freshly adsorbed As dimers in light blue. For the full movie, go to www.fhi-berlin.mpg.de/th/publications/img/ isl-gaas-front.mpg. ${ }^{13}$

At the chosen temperature and pressure conditions we observe island nucleation in the reconstructed surface's trenches. Island growth 
Figure 3. Snapshots from a DFT+kMC simulation of GaAs homoepitaxy. Ga and As substrate atoms appear in green and dark blue, $\mathrm{Ga}$ adatoms in yellow, and freshly adsorbed As dimers in light blue. (a) Ga adatoms preferentially wander around in the trenches. (b) Under the growth conditions used here, an As2 molecule adsorbing on a $\mathrm{Ga}$ adatom in the trench initiates island formation. (c) Growth proceeds into a new atomic layer via $\mathrm{Ga}$ adatoms forming Ga dimers. (d) Eventually, a new layer of arsenic starts to grow, and the island extends itself towards the foreground, while more material attaches along the trench.

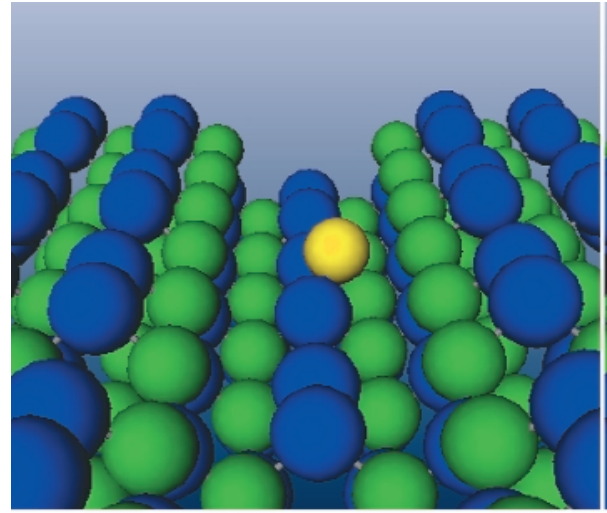

(a)

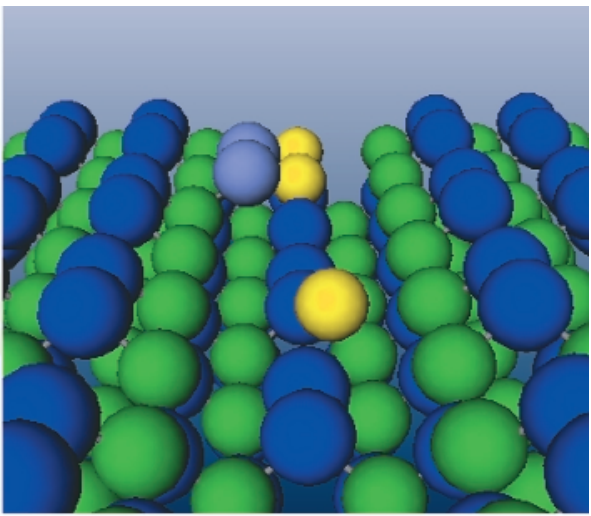

(b)

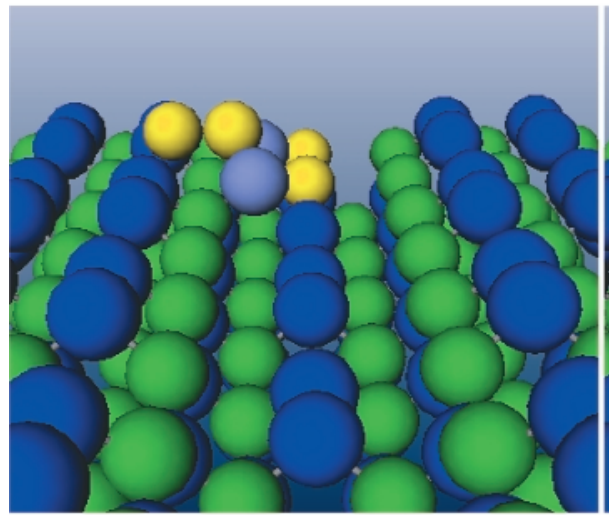

(c)

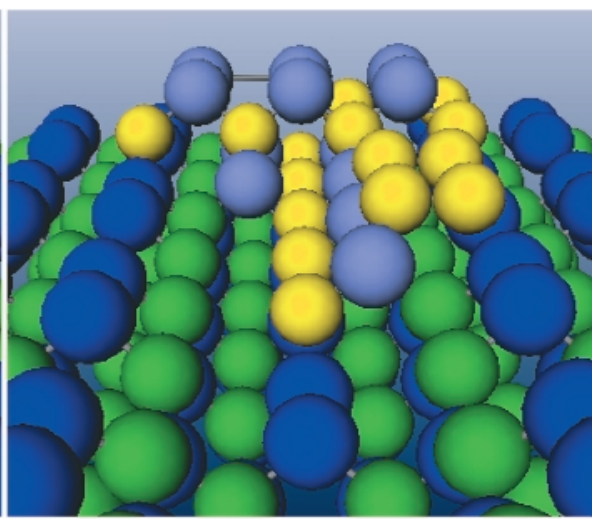

(d) proceeds along the trench, thereby extending into a new layer.

Unlike MBE experiments, these simulations let us study how temperature affects the growth morphology in a wide range and at the atomic level. By varying the temperature used in the simulations, we find that Ga surface diffusion alone causes the island morphology for temperatures up to about $700 \mathrm{~K}$. At higher temperatures, the adsorption of $\mathrm{As}_{2}$ molecules at reactive surface sites becomes reversible, and arsenic losses due to desorption become apparent. The temperature window $\mathrm{MBE}$ crystal growers frequently use permits a compromise between high Ga adatom mobility and stability of As complexes that leads to low island density. ${ }^{13,14}$

\section{Modeling strained heterostructures with thousands of atoms}

For growing GaAs films, crystal growers use, for example, molecular beam epitaxy to deposit gallium (Ga) and $\mathrm{As}^{2}$. With this technique, it is easy to grow heteroepitaxial films by switching the Ga flux at some point to another material, such as indium (In). The mechanical strain re- sulting from the misfit between the substrate and film crystal lattices is often critical to the resulting film morphology, and under certain conditions the spontaneous formation (self-assembly) of quantum dots, consisting of several thousand atoms each, will occur. Here we focus on the methodology to calculate the size and shape of such mesoscopic structures, assuming they are in thermodynamic equilibrium.

A simplified picture of heteroepitaxial growth shows two limiting cases. In the first, the deposited material forms a smooth homogeneously strained film; in the second, the deposited material evolves into 3D islands. Frequently, these islands are pseudomorphic with the substratethat is, the deposited material lattice continues the substrate host lattice without perturbations such as dislocations or grain boundaries being introduced. As a consequence, the islands are elastically strained at their base, where the deposited material is forced to take on the substrate's lattice constant, but the strain is relaxed near the island top, where the atomic layers can take on the generic lattice constant of deposited material, as Figure 4 shows. ${ }^{15}$ This strain relief constitutes the thermodynamic driving force that favors the formation of $3 \mathrm{D}$ islands. On the other hand, the 
tendency to minimize the surface free energy favors the formation of a smooth film.

In fact, real system behavior often lies somewhere between the two limiting cases sketched earlier: A smooth film may form after deposition only up to a certain thickness, and 3D island growth takes over if more material is deposited. This results from an energetic trade-off: while elastic strain relief would favor formation of islands rather than a smooth film, additional surfaces forming at these islands' side facets cost energy.

In a real system, whether and how large islands form depends on the energetic balance between the surface contribution and an elastic contribution from both the islands' bulk and the substrate. Under such conditions, island formation would follow what's known as Stranski-Krastanov growth mode. We've developed an approach to understanding this delicate balance by combining results from the continuum elasticity theory of solids with calculated surface formation energies obtained from density-functional theory.

To illustrate, we discuss a specific example, the formation of small InAs islands after deposition of an InAs film on a GaAs substrate. This combination typically yields islands with a base length between 10 and $20 \mathrm{~nm}$ in the experiment, as Figure 5 shows, and such islands consist of 10,000 to 50,000 atoms each. Most atoms in these islands have a full coordination shell of neighbors and undergo only small relaxations from their bulk positions. Only the surface atoms have a substantially different atomic environment and experience larger displacements due to the surface reconstruction.

Although the elastic relaxations release little energy per bulk atom, bulk elastic effects can be significant-particularly for the largest islands because the elastic energy scales linearly with the number of atoms in the island. Other energetic contributions coming from surface energy changes during island formation counteract the elastic contribution. We compare the total energy of an InAs film (thickness $\Theta_{0}$; see Figure 5, right) with the islands' energy at a given density and a thinner film (thickness $\Theta$; see Figure 5, left). Obviously, both situations must involve equal amounts of material.

To understand the energetics driving island formation, we find it neither necessary nor feasible to employ a full DFT description of all atoms involved but have instead developed a hybrid approach. We write the key quantity, the

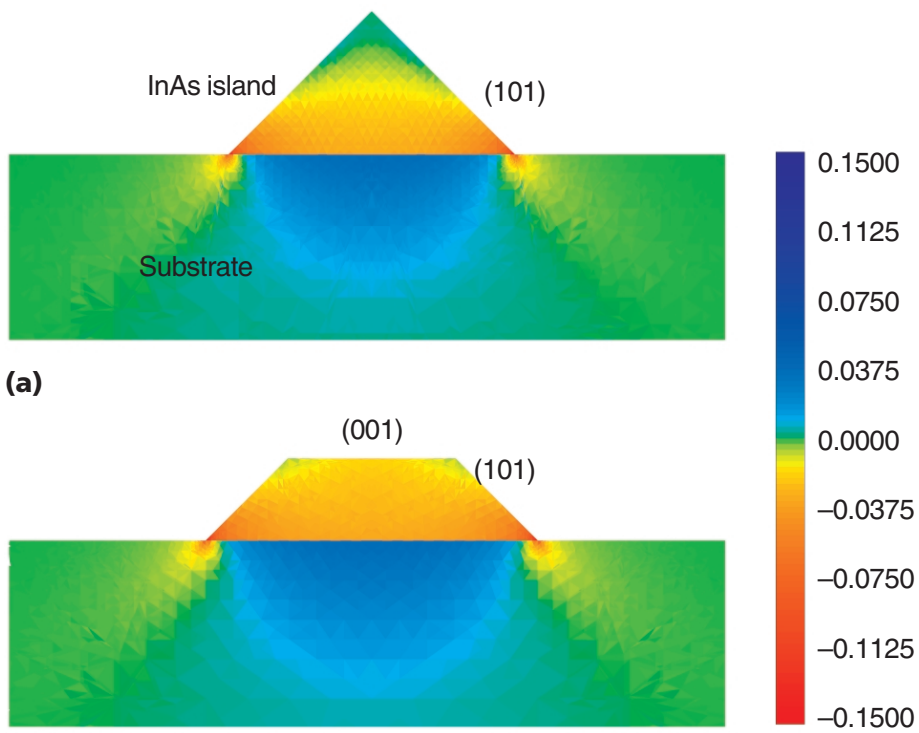

(b)

Figure 4. Strain distribution, as obtained from finite-element calculations ${ }^{15}$ for (a) a pyramidal and (b) a truncated InAs island coherently grown on a $\mathrm{GaAs}(001)$ substrate. The color indicates the trace of the strain tensor on $(010)$ cross sections through the islands.

energy gain per unit volume (or per atom), as a sum of three contributions,

$$
E^{\mathrm{tot}} / V=E^{\mathrm{elast}} / V+E^{\mathrm{surf}} / V+E^{\mathrm{w} 1} / V
$$

where $E^{\text {tot }}$ stands for the total energy gain of an island with volume $V . E^{\text {elast }}$ shows the difference in elastic strain energy between a situation with islands and a homogeneously strained film. We evaluate this quantity, involving small displacements, by numerically solving the elastic continuum equations, using, for instance, the finite-element method. Mechanical engineers regularly use commercially available software for a similar task, calculating strain in structures such as

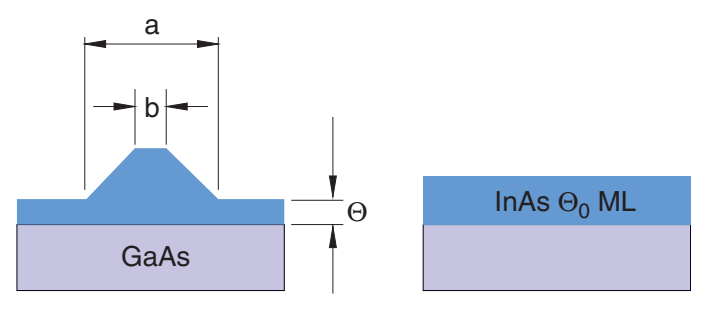

Figure 5. Schematic illustration of the formation of coherent islands on the substrate surface. $\Theta_{0}$ represents the nominal coverage (the total amount of deposited material) and $\Theta$ is the wetting-layer thickness, measured in monolayers (ML). 
bridges and parts of machinery. The extrapolation of this approach to nano-sized structures is justified as long as they consist of several thousands of atoms.

The $E^{\text {surf }}$ and $E^{\mathrm{wl}}$ contributions come from the extra surfaces that islands create and from energetic changes due to thinning of the wetting layer, respectively. DFT calculations, using slab geometry to model one unit mesh of the surface reconstruction, work well for these quantities, which originate from surface bond breaking and distortions. Such calculations require only modest numbers of atoms and are feasible on a modern workstation.

We must, however, perform many calculations to study various surface compositions and reconstructions for each surface orientation. Composition variations make the surface energies, and thus the islands' shape, dependent on the gasphase environment's temperature and pressure. We can retain the elastic effects near the surface that differ from bulk elasticity in Equation 9 if we include contributions to the surface energy linear in the strain $\varepsilon_{j k}$. From DFT slab calculations we obtain surface energies $\gamma^{(i)}$ of the $i$ th facet and the coefficient $\sigma_{j k}^{(i)}$ preceding the linear term, which is unique to surface elastic properties. Likewise, we calculate the formation energy of the wetting layer $\gamma^{\mathrm{wl}}(\Theta)$ within DFT. Thus we designate the surface energy contribution

$$
\begin{aligned}
& E^{\text {surf }}= \\
& \sum_{i}\left(\gamma^{(i)}+\sum_{j k} \sigma_{j k}^{(i)} \varepsilon_{j k}^{(i)}\right) A^{(i)}-\gamma^{w 1}\left(\Theta_{0}\right) A^{0},
\end{aligned}
$$

where $A^{(i)}$ denotes the area of the island's $i$ th side facet, and $A^{0}$ its base area.

Equation 9 also describes the thinning of the wetting layer, defined as

$$
E^{w 1}=\left(1 / n-A^{0}\right)\left[\gamma^{w 1}(\Theta)-\gamma^{w 1}\left(\Theta_{0}\right)\right],
$$

where $\gamma^{\mathrm{wl}}(\Theta)$ is the combined surface and interface energy of the wetting layer, and $n$ is the area density of islands. DFT calculations for thin films show that $\gamma^{\mathrm{wl}}(\Theta)$ is a function of the wetting layer thickness $\Theta$. Only for films thicker than two atomic layers of In does $\gamma^{\mathrm{wl}}(\Theta)$ approach a constant. This has important consequences for the distribution of deposited material between islands and film.

The total-energy expression in Equation 9 lets us quantitatively study various island properties. Once we understand the shape-dependence of the elastic energy and the surface energies, we can systematically study quantum dots' stability as a function of their shape. To this end, we've evaluated the energy gain $E^{\text {relax }}+E^{\text {surf }}$ for a single island, for many trial shapes. Optimizing the shape results in an island with a flat top that displays all low-index side facets. The exact shape depends on the island's size; in particular, the relative importance of the island top's horizontal facet decreases and the island's aspect ratio increases as the islands grow bigger. ${ }^{14}$

The above treatment relied on a thermodynamic argument, following the principle that a given amount of material actually takes on the shape that corresponds to its lowest possible (free) energy. We suggest using the argument with some care, however, as the quantity $E^{\text {tot }} / V$ from Equation 9 is a monotonically decreasing function of $V$. Thus, full thermodynamic equilibrium would correspond to an unrealistic situation where all deposited material has collapsed into a large single island. We can easily remedy this flaw by restricting ourselves to a constrained equilibrium, the constraint being a fixed island density $n$ physically determined by growth conditions during early stages of deposition. Apart from being more physical, this theory lets us obtain information about not only the islands' shape but also their optimum size, once we know the island density and the total amount of deposited InAs. The optimum island size corresponds to a minimum of $E^{\text {tot }} / V$ in Equation 9 when evaluated for fixed values of $n$ and $\Theta_{0}$.

Closely linked to this optimum island size is the remaining thickness $\Theta$ of the wetting layer after the islands develop fully. Only 10 to 30 percent of the total deposited material assembles into islands, depending on the nominal coverage $\Theta_{0}$ and the island density $n$. The results also show that a wetting layer thicker than one monolayer always exists even after three-dimensional island formation has set in. This observation, and the calculated island sizes and aspect ratios, correspond with recent results of scanning-tunneling microscopy investigations.

Our modeling points to a possible method of attaining a desired island size by properly choosing the growth conditions and nominal coverage. ${ }^{16,17}$

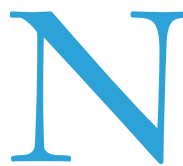
early everything in materials science proceeds from chemical bond formation and dissolution. At surfaces, this typically involves the collective behavior of many atoms, between 10 and 200, which we can effectively calculate using DFT. DFT gives us the total energy or PES on 
which the various atoms move.

Sometimes the minima of this potential-energy surface suffice for calculations, as these minima identify the stable and metastable geometries-that is, the thermal-equilibrium structures. Thermodynamic equilibrium theory does not always apply, however, because many systems are in a local energy minimum. Understanding which of the many PES local minima determine the system's properties requires analyzing the atomic motion along the potential energy surface.

In other words, PES computation is not the final step but forms the basis for description of the atom dynamics. This, in turn, determines the probability of molecular processes (such as chemical reactions) occurring. For the full picture, then, in addition to the knowledge of the PES, we need a description of atomic motion, specifically, how atoms diffuse and what happens when they bump into each other.

Predictive modeling requires distinguishing between significantly more process types than semi-empirical simulations, but we've shown such studies to be feasible. For example, the GaAs growth simulation presented here includes more than 30 process types, each with a clear atomistic, physical meaning. The technique needs further refinement, and we will learn to avoid pitfalls with each new case study. Already, however, first-principles $\mathrm{kMC}$ simulations have provided significant new insight into surface processes and their interplay. si

\section{References}

1. R. Pentcheva et al., "Initial Stages of Heteroepitaxy with Intermixing: $\mathrm{Co}$ on $\mathrm{Cu}(001), "$ to be published.

2. C. Stampfl et al., "Catalysis and Corrosion: The Theoretical Surface-Science Context," to be published in Surface Science, vol. 500, 2001.

3. R. Hughes, "Quantum Computation," Computing in Science \& Eng., vol. 3, no. 2, Mar./Apr. 2001, p. 26.

4. M.R. Sørensen and A.F. Voter, "Temperature-Accelerated Dynamics for Simulation of Infrequent Events," J. Chemical Physics, vol. 112, no. 21, June 2000, pp. 9599-9606.

5. K. Horn and M. Scheffler, eds., Handbook of Surface Science, Vol. 2: Electronic Structure, Elsevier, Amsterdam, 2000.

6. M. Bockstedte et al., "Density-Functional Theory Calculations for Poly-Atomic Systems: Electronic Structure, Static and Elastic Properties and Ab Initio Molecular Dynamics," Computer Physics Comm., vol. 107, nos. 1-3, Dec. 1997, pp. 187-222; www. fhi-berlin.mpg.de/th/fhimd (current 21 Sept. 2001).

7. A. Canning, W. Mannstadt, and A.J. Freeman, "Parallelization of the FLAPW method," Computer Physics Comm., vol. 130, no. 3, Aug. 2000, pp. 233-243.

8. R. Dohmen et al., "A Parallel Implementation of the FP-LAPW Method for Distributed-Memory Machines," Computing in Science \& Eng., vol. 3, no. 4, July/Aug. 2001, pp. 18-29.
9. C. Ratsch, P. Ruggerone, and M. Scheffler, "Density Functional Theory of Surface Diffusion and Epitaxial Growth of Metals," Surface Diffusion: Atomistic and Collective Processes, NATO ASI Series B, vol. 360, 1997, pp. 83-101.

10. K.A. Fichthorn and W.H. Weinberg, "Theoretical Foundations of Dynamical Monte Carlo Simulations," J. Chemical Physics, vol. 95, no. 2, July 1991, pp. 1090-1096.

11. K.A. Fichthorn and M. Scheffler, "Island Nucleation in Thin-Film Epitaxy: A First-Principles Investigation," Physical Rev. Letters, vol. 84, no. 23, June 2000, pp. 5371-5374.

12. K.A. Fichthorn, M.L. Merrick, and M. Scheffler, "A Kinetic Monte Carlo Investigation of Island Nucleation and Growth in Thin-Film Epitaxy in the Presence of Substrate-Mediated Interactions," to be published in Applied Physics A.

13. P. Kratzer and M. Scheffler, "Reaction-Limited Island Nucleation in Molecular Beam Epitaxy of Compound Semiconductors," to be published.

14. P. Kratzer, E. Penev, and M. Scheffler, "First-Principles Studies of Kinetics in Epitaxial Growth of III-V Semiconductors," to be published in Applied Physics A.

15. N. Moll, M. Scheffler, and E. Pehlke, "Influence of Surface Stress on the Equilibrium Shape of Strained Quantum Dots," Physical Rev. B, vol. 58, no. 7, Aug. 1998, pp. 4566-4571.

16. L.G. Wang et al., "Formation and Stability of Self-Assembled Coherent Islands in Highly Mismatched Heteroepitaxy," Physical Rev. Letters, vol. 82, no. 20, May 1999, pp. 4042- 4045.

17. L.G. Wang et al., "Size, Shape, and Stability of InAs Quantum Dots on the GaAs(001) Substrate," Physical Rev. B, vol. 62, no. 3, July 2000, pp. 1897-1904.

For more information on this or any other computing topic, please visit our Digital Library at http://computer. org/publications/dlib.

Peter Kratzer is a computational physicist at the FritzHaber-Institut der Max-Planck-Gesellschaft. He received a PhD in theoretical physics from Technical University Munich in 1993 with a PhD thesis about modeling the dynamics of surface chemical reactions. $\mathrm{He}$ is conducting research in semiconductor materials and nanostructures, and his main interests concern surface chemical reactions and epitaxy of compound materials. Contact him at Fritz-Haber-Institut der MaxPlanck-Gesellschaft, Faradayweg 4-6, D-14195 BerlinDahlem, Germany; kratzer@fhi-berlin.mpg.de; www. fhi-berlin.mpg.de/th/th.html.

Matthias Scheffler is director of the Theory Department of the Fritz-Haber-Institut der Max-Planck-Gesellschaft and a professor at Technical University Berlin. His research concerns condensed-matter theory, materials, and the chemical physics of surfaces. His current interests include developing first-principles methods (using density-functional theory) for molecular simulations that bridge the time and length scales from those of the atomistic processes to those that determine the properties of realistic systems. Contact him at Fritz-Haber-Institut der Max-Planck-Gesellschaft, Faradayweg 4-6, D14195 Berlin-Dahlem, Germany; scheffler@fhi-berlin. mpg.de; www.fhi-berlin.mpg.de/th/th.html. 